\title{
CONNECTIONISM STRATEGY FOR INDUSTRIAL ACCIDENT-ORIENTED EMERGENCY DECISION-MAKING: A SIMULATION STUDY BASED ON PCS MODEL
}

\author{
Lu, J. T. ; Yang, N. D..,**; Ye, J. F. ; Liu, X. G. ${ }^{* * *} \&$ Mahmood, N. \\ ${ }^{*}$ School of Management, Northwestern Polytechnical University, Xi' an 710072, China \\ **Emergency Management Institute, Northwestern Polytechnical University, Xi'an 710072, China \\ ${ }^{* * *}$ College of Economic and Management, Taiyuan University of Technology, Taiyuan 030024, China \\ E-Mail: lut2002@163.com
}

\begin{abstract}
Connectionism strategy is widely used by the policymaker to implement the emergency rescue activities in industrial accidents, exploring its mechanism is helpful to deeply understand the mechanism of behavioural change of policymakers during emergency decision-making procedure. In this study, the emergency process and influential factors as well as three types of emergency disposal schemes, when the connectionism strategy was adopted for rescue activities in coal mining accidents, were qualitatively analysed and concluded. Then, an improved simulation model was proposed based on Parallel Constraint Satisfaction (PCS) model. Lastly an algorithm written in MATLAB was employed to model and explore the influential mechanism of the negative emotion and the concern of policymakers as well as the mode of decision-making tasks on decision time and decision confidence. Results demonstrate that, the higher the policymakers' negative emotion, the shorter the decision time and the higher level of decision confidence they have. Under different modes of decision tasks, the more difficult it is to identify the superiority and inferiority of different disposal schemes, the more decision time the policymakers need and the lower level of decision confidence they have, the decision confidence is more sensitive to the mode of decision-making task, meanwhile the negative emotion and the concern of policymakers have different effects on decision time and decision confidence. Compared with the concern on cues, policymakers' decision confidence is much higher when they focus more on schemes. The study reveals the mechanism of connectionism strategy adopted for emergency rescue activities in industrial accidents, which has a profound significance for optimizing emergency decision support systems for industrial accident management and enhancing the quality of emergency decision-making.

(Received in October 2014, accepted in March 2015. This paper was with the authors 2 months for 1 revision.)
\end{abstract}

Key Words: Industrial Accident, Emergency Management, Coal-mine Accident, Connectionism Strategy, Parallel Constraint Satisfaction (PCS), Decision-making Simulation

\section{INTRODUCTION}

In recent decades, the rapid development of new industrialization and the wide applications of science \& technology have greatly promoted the achievement sharing of industrial civilization in various fields, but the negative effect brought by industrialization has become increasingly prominent. Especially in last fifteen years, industrial accidents have occurred frequently all over the world, such as the 2001 explosion of AZF chemical plant in Toulouse France, Japan's Fukushima nuclear disaster in 2011 and the 8.12 explosion of Ruihui dangerous chemicals warehouse in Tianjin port of China, etc. All of these accidents have also caused a large number of casualties, huge property losses as well as far-reaching social impact. Industrial safety management and emergency decision-making has become a hot topic in academia and industrial circles [1].

After the occurrence of industrial accidents, policymakers need to conduct emergency decision-making among multiple alternative disposal schemes. While the cues influencing the selection of disposal schemes are numerous and each alternative scheme can be supported or 
opposed by several cues, which makes policymakers face a selection dilemma, the above decision mode is called Multiple-Scheme Multiple-Cue (MSMC) decision-making. Furthermore, policymakers in industrial accidents often face the situations with higher time pressure, less cognitive resources, higher environmental complexity and decision-making information deficiency etc., which makes the policymaker can't carry out a thorough rational analysis of available cues and disposal schemes. Studies have shown that, in this case policymakers usually only do their utmost to pursue the cognitive consistency and then make judgements and decisions through the mutual verification between these cues and schemes [2], which is called Connectionism Strategy. Its main mechanism is that if a disposal scheme is supported by more cues, then the possibility of selecting this kind of scheme is higher. While those cues which support the leading scheme will receive more attention, and then the scheme is selected via comprehensive comparison and repeatedly verification [3]. What's more, from the view of decision-making quality, there are two important indicators to evaluate the superiority and inferiority of the selected disposal schemes: One is decision time, the earlier the decision is made, the more time the policymaker has and the lower the occurrence possibility of secondary accidents. The other one is decision confidence, the more confident policymakers feel about their selected disposal schemes, the better the implementation effect is and it can avoid the phenomena of repeated decision-making [4]. From the perspective of the decision-making process, there are three factors which influence the decision quality: The first is policymakers' emotions; policymakers under emergencies often are accompanied by fear, anger, sadness and other negative emotions, which will have a full-scale effect on the cognitive process when making the decision $[5,6]$. The second is the policymakers' concerns, the concern on cues or disposal schemes will lead to different ways of information processing [7]. The last one is the mode of decision-making tasks, which includes information presentation modes, the structure and sequence of cues, the degree of differentiation of disposal schemes etc. [3]. In view of this problem situation, we are proposing the following question: In the situation of industrial accident, how the negative emotion and the concern of policymakers as well as the mode of decision-making tasks influence decision time and decision confidence when the connectionism strategy is adopted for emergency rescue? Addressing this problem can help the policymakers to formulate timelier and more convinced emergency strategy, which can also provide theoretical references for the managers of industrial enterprises to improve the quality of emergency decision-making, and for supervisory departments of industrial safety to formulate corresponding emergency management mechanisms for accident prevention and mitigation.

\section{LITERATURE REVIEW}

Regarding the decision-making strategy of connectionism, plenty of research has been carried out by scholars all over the world. Through a review of existing literatures, we find that there are two main research directions: One is cognitive bias when using connectionism strategy, it is found that this strategy can cause three kinds of effects, the first is confirmation bias in the procedure of searching for cues, it tends to continue to search for cues which support the dominant scheme and ignore the cues that do not support the dominant scheme, this effect often occurs in the field of judicial trials and leads to a wrong judgment [8]. The second is coherence shift during the process of cue evaluation, namely policymakers often overestimate the importance of cues which support the dominant scheme while underestimate the importance of cues that don't support the dominant scheme. This kind of shift exists during three stages in emergency decision-making: before, during and after, and which is also is a common pitfall in the process of emergency medical rescue [9]. The third is primacy effect when selecting disposal schemes, the scheme supported by cues at first will be easily pre- 
occupied and recognized by policymakers, and also the scheme of this kind tends to be selected as the final disposal measure even it is suboptimal [10]. The other is the studies on influential factors of connectionism strategy. First of all, with regard to policymakers' emotion, some studies found that, compared with the positive emotions, policymakers with negative emotions tended to show more confirmatory biases in order to reduce cognitive dissonance when they are searching cues [11]. At the same time, negative emotions can reinforce the degree of coherence shift, and cues that are beneficial to maintain emotion are considered more important [12]. Secondly, in term of policymakers' concerns, if policymakers pay more attention to disposal schemes, it will increase the degree of coherence shift, while if they focus on cues, it will decrease the degree of coherence shift [13]. Meanwhile the policymaker will show a higher degree of confirmation bias if they pay more attention to the dominant scheme rather than the cues which support the dominant scheme [14]. Finally, on the aspect of the mode of decision-making tasks, results from Bergert \& Nosofsky's study suggested that, the policymakers were inclined to adopt connectionism strategy if it was more difficult using the involved cues to distinguish the superiority and inferiority of different alternative disposal schemes [15]. Additionally, if the decision-making tasks contain more cues and disposal schemes and the relationships among them are more complex, the policymakers will be more apt to adopt connectionism strategy [2].

It can be seen that the existing literature has deeply explored the connectionism strategy and its influential factors under the particular decision-making environment, even though research of this kind has rarely focused on the variation law analysis of decision time and decision confidence which represent the decision quality when policymakers are using connectionism strategy, and most of them concentrate on the fields of consumer behaviour, medical diagnosis and judicial decision. But they still have important reference value for the research on emergency management in industrial accident. It is also worth mentioning that, Glockner and his co-authors found that Parallel Constraint Satisfaction (PCS) model can well describe the decision-making mechanism of connectionism strategy, they also carried out a series of simulation experiments by using this model to preliminarily explore the variation law analysis of decision time and decision confidence [16-18]. Nevertheless their studies are lack of further discussion about the influence of the negative emotion and the concern of policymakers as well as the mode of decision-making tasks on decision time and decision confidence, and its influential mechanism is also unclear. In order to cover the shortage of previous studies, the below two issues will be analysed in this study: (1) Combing with indepth interview and questionnaire survey data from managers with different ranks of a mining company located in Hebei province of China, the emergency process, influential factors as well as the types of emergency disposal schemes, when the connectionism strategy is adopted for emergency rescue by the policymakers, are analysed and concluded qualitatively from a theoretical perspective; (2) Based on the study of issue (1), starting from theoretical modelling, the PCS model is applied to emergency decision-making in the industrial accident situation, the corresponding simulation model is constructed, and then a computer simulation method in MATLAB environment is employed to explore the influential mechanism of the negative emotion and the concern of policymakers as well as the mode of decision-making tasks on decision time and decision confidence, hoping the relevant results can serve as a supplementary analysis of contents which are not discussed in details in issue (1).

\section{CONNECTIONISM STRATEGY FOR INDUSTRIAL ACCIDENT- ORIENTED EMERGENCY DECISION-MAKING}

Considering the coal mining accident (such as gas explosion, water-inrushing, coal dust explosion, etc.) is a typical industrial accident in China's energy industry fields, so combined 
with the emergency decision-making situation described in the article, we carried out a questionnaire survey at a mining company which is located in Hebei province of China between May $25^{\text {th }} 2014$ and June $20^{\text {th }} 2014$. After getting the approval from respondents, an in-depth interview was also conducted with a small selected group of participants (2 middlelevel managers, 2 lower-level managers and 4 front-line miners). In order to ensure the interview quality as well as in response to the respondents' request, we promised not to disclose the respondents' privacy to any of the third-party, the key information of their privacies would be anonymous and all the interview data were only used for academic research. Meanwhile, in order to discover the real viewpoints and opinions of respondents, we tried to choose informal occasions or enclosed environments to proceed the interview with the respondents separately, so that the external factors, which interfered with the expression of respondents' real intention, can be excluded.

\subsection{Emergency decision-making process in the coal mining accident when using connectionism strategy}

When the coal mining accident occurs, policymakers or emergency planners can't accurately grasp the whole sequence of events at the first moment, and carry out a thorough analysis on all the obtained cues and disposal schemes. To avoid the long-time hesitations among different schemes, during the stage of information processing, policymakers are inclined to continuously confirm that a certain preferred scheme has a higher feasibility [9]. Additionally, the more experiences of emergency rescue the policymakers have, the stronger the preference, which will further urge the policymakers to make decisions using connectionism strategy. This decision-making process is shown in Fig. 1.

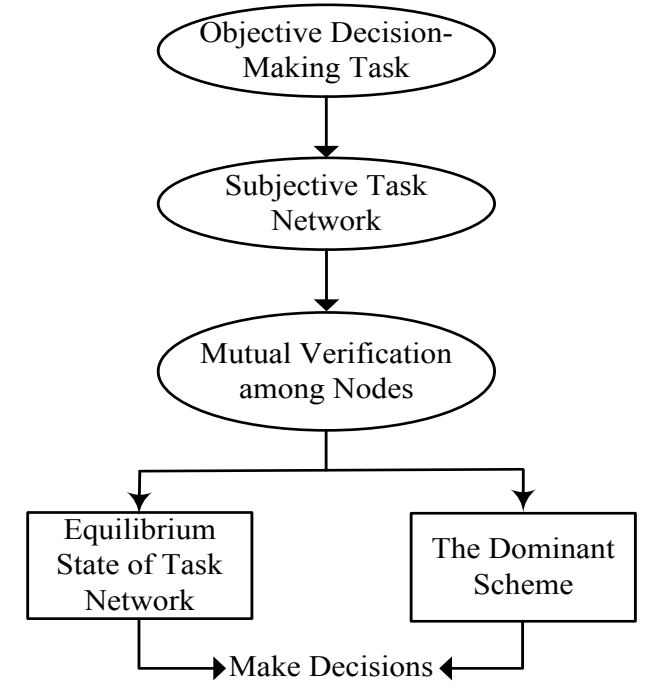

Figure 1: Emergency decision-making process in the coal accident using connectionism strategy.

During the initial stage of emergency decision-making, policymakers are facing multisources information which is even conflicting. At this time they examine the coal mining accident from an overall perspective instead of partially analysing a certain aspect of the accident. Therefore the policymaker will try to perform a repeated procedure of cognitive processing to analyse the scattered information, using the associative method to perfect the missing information involved in the accident, and then a structured scenario, which is consistent with the assumed accident scene, is constructed subjectively [3]. Namely, the policymakers will consider the coal mining accident they are facing as a type of network structure to construct a subjective task network, in which, each alternative disposal scheme or clue is identified as a network node, all the nodes are interconnected and interacted [19]. 
On the basis of the construction of subjective task network, driven by the motivation of cognitive consistency, policymakers consistently seek to understand the coal mining accident and decision-making tasks they are facing via mutual-verification among different nodes. Furthermore, not only the cue can influence the evaluation of disposal schemes, but also the disposal scheme can affect cues [20]. If a disposal scheme is supported by more important cues, it indicates that the activation degree of this scheme is higher. On the contrary, the higher the feasibility of the disposal scheme, the higher the activation degree of cues which support the disposal scheme. The higher activation degree of a certain disposal scheme suggests that this scheme is more feasible, for cues it is evaluated using the same rule. This is a dynamic adjustment process; policymakers complete this process within a short time through a series of iterative steps in the heuristic-cognitive system to make the task network reach an equilibrium state [21].

During the above dynamic adjustment process, policymakers select a dominant scheme in the task network, then gradually increase the preference for it, and finally subjectively increase the credibility and importance of the cues that support the selected dominant scheme. When the task network reaches the equilibrium state, the decision-making task is cognitively processed as a mental representation which consistently supports the selected dominant scheme, and the policymakers finally put the selected dominant scheme into emergency rescue activities in industrial accidents when their cognitive consistency levels reach a certain threshold [18]. Adopting the connectionism strategy for emergency decision-making in the coal mining accident can help the policymakers to form the preference for the selected dominant scheme in a short period without respectively analysing each disposal scheme, which result in shorter decision time and a higher decision confidence [9].

\subsection{Influential factors of using connectionism strategy}

According to the analysis of in-depth interview and questionnaire survey data, we found that the degree of using connectionism strategy under different modes of decision-making tasks was different, the policymakers' decision time and decision confidence also showed a certain differentiation, and the degree of using connectionism strategy was influenced by the following three factors:

(1) Negative emotion of policymakers

When the coal mining accident occurs, policymakers are often accompanied by stronger negative emotions (fear, anger, sadness etc.), which makes them have a higher level of motivation of emotion maintenance, namely try to keep positive emotions and reduce negative emotions [22]. While the inconsistency and conflict among nodes in the task network can further increase negative emotions via cognitive dissonance. To avoid this situation, policymakers will frequently use connectionism strategy for emergency decision-making [11].

(2) Concern of policymakers

Owing to the diversity of policymakers' cognitive style and the presentation mode of decision-making tasks, the policymakers' selections that they concentrate more on disposal schemes or cues show different trends [23]. When paying more attention to the schemes, what they mainly consider is that "which cues will support/not support the schemes", and then during the process of mutual verification, the influence of cues on schemes is greater. By contrast, when focusing more on the cues, the effect of schemes on cues is greater $[13,14]$.

(3) Mode of decision-making tasks

With respect to different kinds of emergency decision-making tasks, the influential relationships between each disposal scheme and cue are different. So if the difference in the quality and quantity of the cues that support different disposal schemes is not significant, it is more difficult for policymakers to identify the dominant scheme from multiple alternative disposal schemes, and their preferences for the potential dominant scheme are strengthened in 
the follow-up procedure [24]. In this case, although it is more necessary for policymakers to adopt connectionism strategy, the decision time and decision confidence shown in the emergency rescue procedure are not ideal.

\subsection{Types of emergency disposal schemes when using connectionism strategy}

Through the coding and structural analysis on the in-depth interview and questionnaire survey data using Altas.ti 5.0 software, we summed up two types of emergency behaviour shown as follows: (1) After the coal mining accident, due to being afraid of a greater harm caused by the secondary accident, during the initial stage of emergency rescue, policymakers are inclined to conservatively ground-initiated rescue mode, including the trapped miners' behaviours of waiting for rescue in the mine refuge chamber and self-rescue/mutual aid in the mine when the ground rescue forces can't reach underground coal mines in time owing to the limitation of various objective conditions, so as to reduce the risk in decision-making and increase the safety coefficient of emergency rescue; (2) Being faced with the tragic accident scene, all the individuals at the scene of emergency rescue are accompanied by different degrees of negative emotions such as fear, anger and sadness etc., the pressure from the rescue commitment to the public made by the policymakers as well as the reputation seeking and maintenance motivation of policymakers themselves can strengthen the atmosphere of "rapid rescue response" $[25,26]$, which then makes the rescue behaviour at the present time deviate from the established contingency plans to a certain extent, resulting in irrational decision-making and emergency rescue. The survey \& questionnaire data also show that there are three types of emergency disposal schemes which are involved with the emergency behaviour mentioned above [6], from the perspective of risky decision-making, it can be summarized as: (1) Risk-seeking; (2) Risk-aversion; (3) Risk-neutralness.

\section{CONSTRUCTION OF SIMULATION MODEL BASED ON PARALLEL CONSTRAINT SATISFACTION}

For the policymakers at the scene of emergency rescue, under different types of negative emotions, concerns and modes of decision-making tasks, the decision process of using connectionism strategy is different, so the decision time and decision confidence also show a certain difference. To deeply explore the influential mechanism, on the basis of the relevant studies from Glöckner and Hodges [17] as well as parallel constraint satisfaction theory, an improved simulation model is established in this paper to analyse the proposed problem.

To meet the need of our study, the coal mining accident described above is simplified as a MSMC decision-making task which includes three types of alternative disposal schemes, and then it is converted into a subjective task network by the policymakers (see Fig. 2). In which, we assume that the cues are independent of each other, $a_{C i}$ represents the $i^{\text {th }}$ cue, $a_{O j}$ stands for the $j^{\text {th }}$ scheme, in addition, there exists a general validity node $a_{g}$.

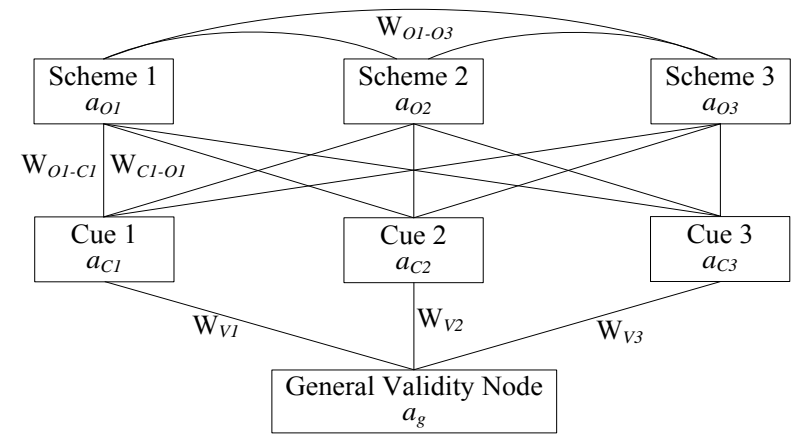

Figure 2: Diagram of the subjective task network. 
As can be seen from Fig. 2, there are different degrees of mutual influence among these nodes, in which, $W_{C i-O j}$ denotes the influence of cue $a_{C i}$ on scheme $a_{O j}$, which is determined by the modes of decision-making tasks. If scheme $a_{O j}$ is supported by cue $a_{C i}$, then $W_{C i-O j}>0$, otherwise $W_{C i-O j}<0$. At the same time, scheme $a_{O j}$ also has a reverse effect on cue $a_{C i}$, which can be expressed as $W_{O j-C i}$, and it also satisfies $W_{O j-C i}=W_{C i-O j}$. $W_{O i-O j}$ is used to describe the mutual effect of different schemes, we also define $W_{O i-O j}<0$ to express the mutual inhibitory effect among different schemes. Lastly $W_{v i}$ is employed to describe the mutual effect between the general validity node $a_{g}$ and cue $a_{C i}$, which also represents the importance of cue $a_{C i}$ and its value is determined by the validity $v_{i}$ of cue $a_{C i}, W_{v i}=v_{i}{ }^{2} / 100$. In this study we set $W_{v i}$ is $0.9,0.8$ and 0.7 respectively. Additionally, cue validity is the probability which indicates that if the cue theoretically shows that a certain scheme is better than others and the scheme is actually proved to be optimal.

In the decision-making task mentioned above, each node is activated at different levels in the minds of policymakers. The higher the activation degree of a certain disposal scheme or cue is, the more important it is for policymakers. We use $a_{i}(t)$ to express the activation degree of the $i^{\text {th }}$ node at time $t$. In the subsequent process, $a_{i}(t)$ is iterated according to the algorithm described in Eq. (1), the iterative process is stopped when reaching the equilibrium condition, namely for all nodes, they satisfy $a_{i}(t+1)-a_{i}(t)<10^{-2}$ [3]. In view of the factors of the negative emotion and the concern on cues or schemes, on the basis of PCS model, we propose an improved iterative model as below:

$$
\left\{\begin{array}{l}
a_{i}(t+1)=a_{i}(t)(1-\text { decay })+ \begin{cases}\text { input }_{i}(t) \times\left(a_{i}(t)-\text { floor }\right) \times E & \left(\text { input }_{i}(t)<0\right) \\
\text { input }_{i}(t) \times\left(\text { ceiling }^{\prime} a_{i}(t)\right) \times E & \left(\text { input }_{i}(t)>0\right)\end{cases} \\
\text { input }_{i}(t)=\sum_{j=1 \rightarrow n} R_{C} \times R_{O} \times W_{j i} a_{i}(t)
\end{array}\right.
$$

In Eq. (1), decay is attenuation factor which expresses the natural attenuation of the activation degree of nodes over time, ceiling and floor refer to the maximum and minimum values of the activation degree of nodes respectively, let ceiling $=1$ and floor $=-1$ be the constants in this study. Meanwhile, according to the opinion of McClelland and Rumelhart [21], the activation degree of each node has nothing to do with its initial activation degree when the task network reaches the equilibrium state. Thus at the beginning of the decisionmaking task, we set the values of the activation degree of all nodes to 1 . Parameter $E$ is the intensity of negative emotion, the higher the negative emotion, the greater the total input derived from other nodes which influence the activation degree of the present node. Parameters $R_{c}$ and $R_{o}$ respectively represent the degree of policymakers' concern on cues or schemes, a higher value of $R_{c}$ indicates schemes has a greater influence on cues, a higher value of $R_{o}$ indicates cues has a greater influence on schemes. It should be pointed out that, for any value of $W_{C i-O j}, R_{c}$ is 1 , while for $W_{O j-C i}, R_{o}$ is $1 . E, R_{c}$ and $R_{o}$ are considered as independent variables in the simulation process. In addition, when the task network reaches the equilibrium state, we use its experienced iteration number to describe decision time which is noted as $T F$. The difference of the activation degree of the two schemes which are listed in the top 2 activation degrees is employed to define decision confidence that is marked as $C F D$. The higher $C F D$ indicates that policymakers are more convinced about the selected dominant scheme is superior to other schemes.

According to the above analysis, combined with the need of the research problems and simulation requests in our study, all the parameter settings in the proposed model are provided in Table I. 
Table I: Parameter settings of the simulation model.

\begin{tabular}{|c|c|l|c|}
\hline & Parameter & \multicolumn{1}{|c|}{ Definition } & Value \\
\hline \multirow{4}{*}{ Constant } & decay & Attenuation factor & 0.05 \\
\cline { 2 - 4 } & $W_{O i^{-} O} O_{j}$ & Influence coefficient among schemes & -0.02 \\
\cline { 2 - 4 } & Ceiling / Floor & $\begin{array}{l}\text { The maximum and minimum values of the } \\
\text { activation degree }\end{array}$ & $1 /-1$ \\
\cline { 2 - 4 } & $W_{C^{-} O_{j}} / W_{O i^{-} C j}$ & Influence coefficient between schemes and cues & -0.01 or 0.01 \\
\cline { 2 - 4 } & $W_{v i}$ & Influence coefficient between cues and $a_{g}$ & $W_{v i}=v_{i}^{2} / 100$ \\
\hline \multirow{4}{*}{$\begin{array}{c}\text { Dependent } \\
\text { variable }\end{array}$} & $T F$ & Decision time & iteration number \\
\cline { 2 - 4 } & $C F D$ & Decision confidence & $\begin{array}{c}\text { Difference of the } \\
\text { activation degree } \\
\text { of schemes }\end{array}$ \\
\hline \multirow{2}{*}{$\begin{array}{c}\text { Independent } \\
\text { variable }\end{array}$} & $E$ & Negative emotion factor & $E \geq 1$ \\
\cline { 2 - 4 } & $R_{o}$ & Concern on schemes factor & $R_{o} \geq 1$ \\
\cline { 2 - 4 } & $R_{C}$ & Concern on cues factor & $R_{C} \geq 1$ \\
\hline
\end{tabular}

Lastly, as shown in Table II, three representative modes of decision-making tasks are analysed comparatively. Wherein, each cue is ranked in accordance with its cue validity in descending order. The symbol "+" represents the promotion effect existed between cues and schemes, correspondingly $W_{C i-O j}=W_{O j-C i}=0.01$. The symbol "_" stands for the inhibition effect existed between cues and schemes, correspondingly $W_{C i-O j}=W_{O j-C i}=-0.01$, while symbol "/" indicates that there is no relationship between cues and schemes. So it is not difficult to find that, from the view of the difficulty of distinguishing different disposal schemes, the difficulty level of Mode $1<$ the difficulty level of Mode $2<$ the difficulty level of Mode 3.

Table II: Three representative modes of decision-making tasks in the simulation model.

\begin{tabular}{|l|c|c|c|c|c|c|c|c|c|}
\hline & \multicolumn{4}{|c|}{ Mode 1 } & \multicolumn{3}{c|}{ Mode 2 } & \multicolumn{3}{c|}{ Mode 3 } \\
\cline { 2 - 10 } & Scheme & Scheme & Scheme & Scheme & Scheme & Scheme & Scheme & Scheme & Scheme \\
& 1 & 2 & 3 & 1 & 2 & 3 & 1 & 2 & 3 \\
\hline Cue 1 & + & - & $/$ & + & - & - & + & - & + \\
\hline Cue 2 & + & + & - & + & + & - & + & + & - \\
\hline Cue 3 & - & $/$ & + & - & + & + & - & + & + \\
\hline
\end{tabular}

\section{SIMULATION \& RESULT ANALYSIS BASED ON MATLAB}

\subsection{Influence of the negative emotion and the mode of decision-making tasks on decision time and decision confidence}

Under the condition that the policymakers' concerns are not considered (Let $R_{c}=1, R_{o}=1$ ), Fig. 3 shows the influence of the negative emotion and different modes of decision-making tasks on decision time and decision confidence, wherein, horizontal axis represents the intensity of negative emotion $E$, longitudinal axis in Fig. 3 a refers to decision time $T F$, longitudinal axis in Fig. 3 b denotes decision confidence $C F D$.

As can be seen from Fig. 3, with the gradual increase of the intensity of negative emotion $E$, the decision time $T F$, that the task subjective network of policymakers needs to reach the equilibrium state, shows an overall decreasing trend, while the decision confidence $C F D$ displays a general upward tendency. The above results demonstrate that policymakers' negative emotion in the coal mining accident can shorten the decision time and enhance the decision confidence. 


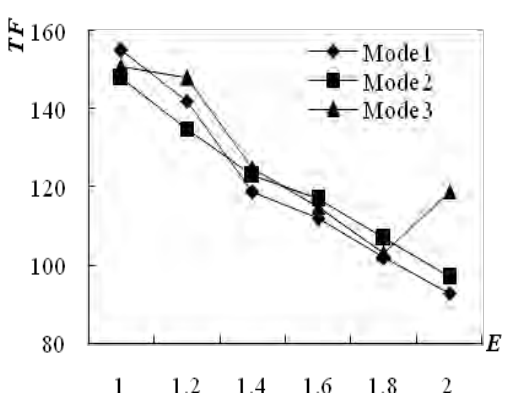

a)

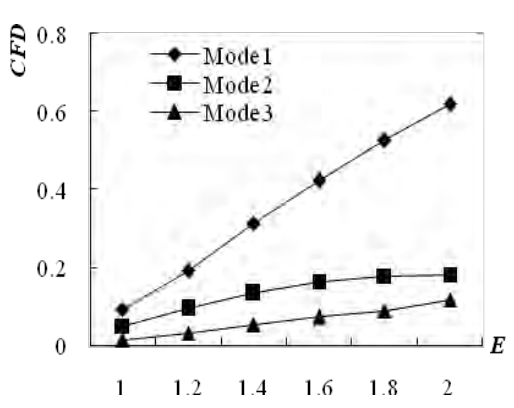

b)

Figure 3: Influence of the negative emotion and the mode of decision-making tasks on decision time and decision confidence.

It should be pointed out that, the decision time $T F$ under different modes of decisionmaking tasks illustrates a certain degree of difference. On the whole, $T F$ of Mode $1<T F$ of Mode $2<T F$ of Mode 3. While the decision confidence $C F D$ under different modes of decision-making tasks displays a significant difference, $C F D$ of Mode $1>C F D$ of Mode $2>$ $C F D$ of Mode 3. This indicates that the more difficult it is to distinguish the superiority and inferiority of different alternative disposal schemes, the more decision time the policymakers need, along with a lower level of decision confidence. Decision confidence is more sensitive to the mode of decision-making task than decision time.

Additionally, there exists an interaction effect between the negative emotion and the mode of decision-making task. Compared with the other two modes, in mode 1, with the gradual increase of the intensity of negative emotion $E$, the descent range of decision time and the increase range of decision confidence are much higher. So according to the obtained results above, the more difficult it is to distinguish different alternative disposal schemes in the present decision task, the less influence the negative emotion has on decision time and decision confidence.

\subsection{Influence of the concern of policymakers on decision time and decision confidence in different modes of decision-making tasks}

Under the case that doesn't consider policymakers' negative emotion (Let $E=1$ ), the influence of the concern of policymakers on decision time and decision confidence in different modes of decision-making tasks is illustrated in Fig. 4, in which, for the curve of concern on cues, horizontal axis represents $R_{c}$, while with respect to the curve of concern on schemes, horizontal axis stands for $R_{o}$, and longitudinal axis in Figs. 4 a, c, e refers to decision time $T F$, longitudinal axis in Figs. $4 \mathrm{~b}, \mathrm{~d}$, f denotes decision confidence $C F D$. The simulation results from Figs. $4 \mathrm{a}$ and b, Figs. $4 \mathrm{c}$ and $\mathrm{d}$ as well as Figs. $4 \mathrm{e}$ and $\mathrm{f}$ were calculated under the corresponding task mode 1,2 and 3 respectively.

As seen from Figs. 4 a, c, e, for one thing, with the increase of $R_{c}$ and $R_{o}$, the value of $T F$ presents an increasing trend after the first decrease, which indicates that policymakers' concerns on cues/schemes can effectively the decision time, but when the concern of this kind increases to a certain level, it can conversely prolong the decision time. For another thing, with the increase of the degree of policymakers' concern in mode 1 , the decision time in the case of the policymakers concentrate on schemes is much longer than that in the case of the policymakers concentrate on cues, this difference in mode 2 is reduced to some extent. While in mode 3, with the increase of the degree of policymakers' concern, the decision time when policymakers focus on cues is much longer than that when policymakers focus on schemes. It is shown that, compared with concern on cues, the more complex the decision-making tasks are, and the more difficult it is to distinguish the superiority and inferiority of different 
schemes, the shorter decision time the policymakers need to deal with the coal mining accident when they concentrate on schemes.

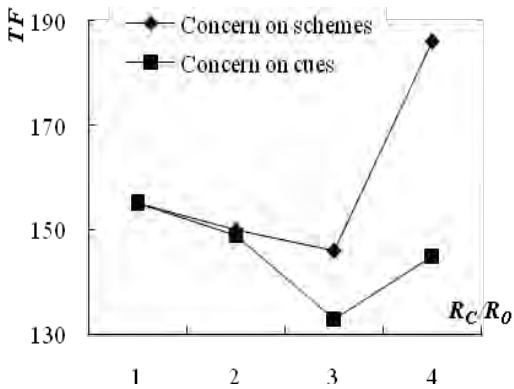

a)

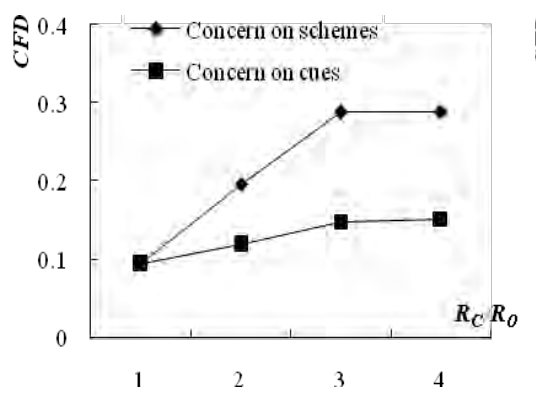

b)

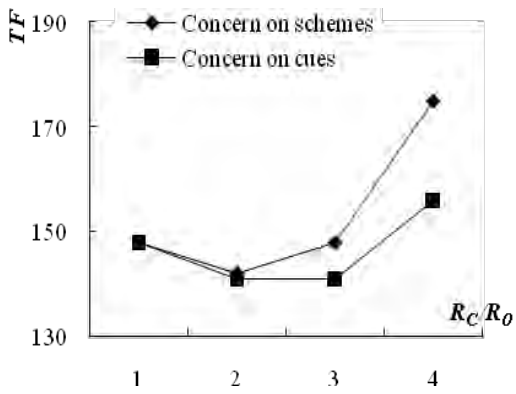

c)

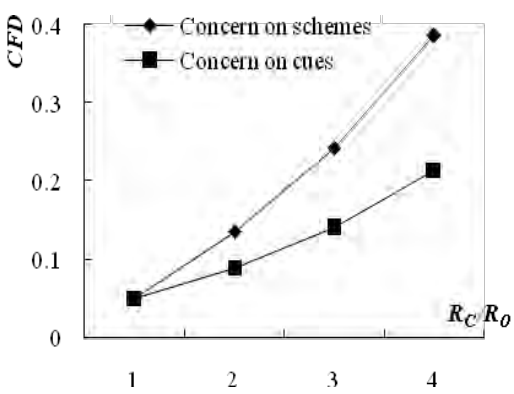

d)

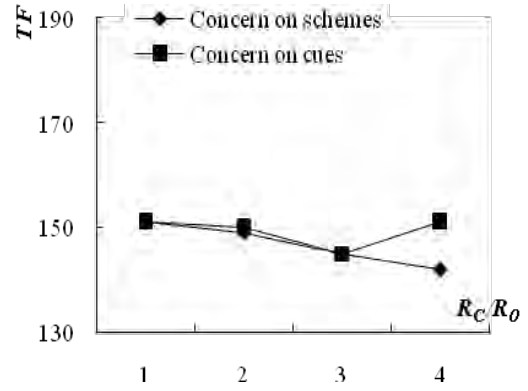

e)

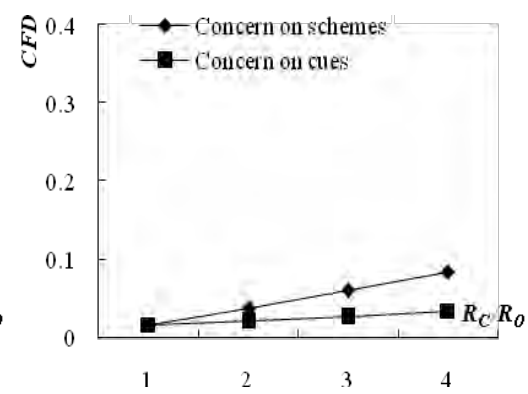

f)

Figure 4: Influence of the concern of policymakers on decision time and decision confidence under different task modes.

Results from Figs. $4 \mathrm{~b}, \mathrm{~d}$, f show that, firstly, with the increase of $R_{c}$ and $R_{o}$, the value of $C F D$ in each figure is increasing, which indicates that the enhancement of the degree of policymakers' concern on cues or schemes can eventually improve the decision confidence. Secondly, compared with concern on cues, policymakers' decision confidence is much higher when they focus more on schemes, and the decision confidence is more sensitive to the degree of policymakers' concern. This demonstrates that when policymakers pay more attention to disposal schemes, they are inclined to adopt connectionism strategy. Lastly, in mode 2, the degree of policymakers' concern has a higher impact on decision confidence, the difference of the mentioned impact is most significant between the concern on cues and schemes, the trend of this kind of difference is not obvious in mode 1 , and there is no clear difference existed in mode 3. It is shown that, when the difficulty of distinguishing different disposal schemes is at the intermediate level, the enhancement of the degree of policymakers' concern on cues or schemes can highly improve their decision confidence.

\section{DISCUSSION}

(1) The influence of negative emotion of policymakers on decision time

Simulation results show that, the higher the policymakers' negative emotion, the shorter the decision time they need to deal with the industrial accident, which is consistent with the conclusion of references [11] and [27]. In addition to the motivation to reduce policymakers' cognitive dissonance and improve policymakers' emotion [28], another possible reason is that, owing to the relative decrease of cognitive resources of policymakers with negative emotions as well as the decline of their cognitive abilities, policymakers can't accurately analyse the validity of each cue and their influences on schemes, so they tend to use connectionism strategy to deal with the coal mining accident, which can also more easily make policymakers form the attitude to agree with the dominant scheme and lead to a shorter decision time. This 
is also confirmed by our survey, such as most respondents considered that, "when the coal mining accident occurred, everyone was very anxious, in order to save the trapped miners as soon as possible, the rescue work was not completely conducted according to the established contingency plans."

(2) The influence of negative emotion of policymakers on decision confidence

As can be seen from the result of Fig. $3 \mathrm{~b}$, policymakers' negative emotion can enhance their decision confidence; this is just in contradiction with the conclusion of reference [4]. In our view it is because that, there may be two types of influence mechanisms of negative emotion on decision confidence, namely, besides the connectionism strategy analysed above, the other is emotion-congruency effect, policymakers with negative emotions are inclined to make more pessimistic judgments which are in agreement with the present emotional state [29]. While the simulation model in this study only reflects the first mechanism, as per the analysis of questionnaire survey data [6], for the emergency decision-making in real accident situations, the performance of the second mechanism is better than the first one.

(3) The influence of the mode of decision-making tasks on decision confidence and decision time

Simulation result from Fig. 3 shows that, under different task modes, the more difficult it is to distinguish different alternative disposal schemes, the more decision time the policymakers need and the lower level of decision confidence they have. This is in accordance with the conclusion obtained from reference [17]. In our opinion, if the discernibility among different schemes is lower, it is more difficult to make the difference of the activation degree of these schemes reach the decision confidence threshold, which also needs more iteration numbers. Meanwhile, the more difficult it is to identify different alternative schemes, the less influence the negative emotion has on decision time and decision confidence. Because even though negative emotion can strengthen the PCS process, its impact is also constrained by other factors including the mode of decision-making task itself. In our survey mentioned above, lower-level managers and front-line miners generally considered that, "owing to the unexpectedness and unpreparedness of the coal mining accident as well as the changeability of the disposal situation, some aspects of the established contingency plans were appropriately adjusted according to the requirement of rescue work, so as to obtain multiple alternative disposal schemes for emergency decision-making, which also simultaneously leads to a higher level of time pressure to rescuing work."

(4) The influence of the concern of policymakers on decision time

According to Figs. 4 a, c and e, we can find that, with the increase of the degree of policymakers' concern on cues or schemes, the decision time illustrates an increasing trend after the first decrease. The reason, in our view, is that when the degree of policymakers' concern is at the intermediate level, the increase of $R_{c}$ and $R_{o}$ can speed up the PCS process, making the task network reach the equilibrium state in a shorter time. But when $R_{o}$ exceeds a certain value, on the contrary it makes each scheme have a higher activation degree, each cue have a lower activation degree and policymakers' overall recognition of the task network in the state of disequilibrium and instability, which finally increases the cognitive difficulty, as a result, the task network needs more iteration numbers to reach a new equilibrium state. This conclusion is verified by our investigation, "the existence of the phenomenon of an overresponse to emergency rescue induced an interference effect on the rescue work to a certain degree."

(5) The influence of the concern of policymakers on decision confidence

It can be seen from the simulation result of Figs. $4 \mathrm{~b}$, $d$ and $f$, compared with policymakers' concern on cues, their decision confidence is much higher when they concentrate more on schemes, the result is in line with the findings obtained from references [13] and [14]. We argue that since the decision confidence is determined by the difference of 
the activation degree of different schemes. When the policymakers focus more on schemes, the increase of $R_{o}$ can strengthen the impact of cues on schemes, which also directly amplifies the difference of the activation degree of different schemes. By contrast, when the policymakers pay more attention to cues, the increase of $R_{c}$ can enhance the effect of schemes on cues, which finally indirectly influence $C F D$ through enlarging the difference of the activation degree of different schemes. This explanation is also supported by the respondents' views during our survey, "no matter whether supervisory departments, mining enterprises, or public opinion, the major aspect they focused on was how to successfully complete the rescue task through feasible and effective rescue measures."

\section{CONCLUSION}

After the occurrence of industrial accidents, the rescue work is typically accompanied by higher levels of complexity and uncertainty as well as significant continuing personal pressure, policymakers are inclined to analyse the decision task from an overall perspective and use connectionism strategy to deal with the coal mining accident. Given this problem situation, combined with the questionnaire survey and in-depth interview carried out at one mining company which is located in Hebei province of China, the emergency process, influential factors as well as the types of emergency disposal schemes, when the connectionism strategy was adopted for emergency rescue by the policymakers, were qualitatively analysed and concluded. Then the corresponding simulation model was proposed on the basis of PCS model. Lastly an algorithm written in MATLAB was employed to model and explore the influential mechanism of the negative emotion and the concern of policymakers as well as the mode of decision-making tasks on decision time and decision confidence, the following conclusions can be drawn: (1) The higher the policymakers' negative emotion, the shorter the decision time they need to deal with the industrial accident and the higher level of decision confidence they have; (2) Under different modes of decision-making tasks, the more difficult it is to identify the superiority and inferiority of different alternative disposal schemes, the more decision time the policymakers need and the lower level of decision confidence they have. Meanwhile decision confidence is more sensitive to the mode of decision-making task; (3) Compared with the concern on cues, policymakers' decision confidence is much higher when they focus more on schemes, and the decision confidence is more sensitive to the degree of policymakers' concern; (4) Under different modes of decision tasks, the more difficult it is to distinguish the superiority and inferiority of different disposal schemes, the less influence the negative emotion has on shortening decision time and enhancing decision confidence; (5) With the increase of the degree of policymakers' concern on cues or schemes, the decision time firstly illustrates a decreasing trend, but when the degree of policymakers' concern exceed a certain value (threshold), the decision time then increases significantly; (6) Under different modes of decision tasks, when the difficulty of distinguishing different disposal schemes is at the intermediate level, the increase of the degree of policymakers' concern on cues or schemes can obviously enhance their decision confidence.

Nonetheless, the study also suffers some limitations. For one thing, the survey and indepth interview are only carried out in mining enterprises, thus the representativeness of research samples and the universality of the obtained conclusions show certain limitations. For another thing, we only add two variables (i.e. negative emotion and concern on cues/schemes) in the proposed simulation model, while the difference of decision-making behaviours is not merely caused by the above two factors. Therefore, how to combine other behavioural decision models to better solve industrial emergency management problems, optimize emergency decision support systems for industrial accident management and enhance the quality of emergency decision-making, is still worthy of further study. 


\section{ACKNOWLEDGEMENT}

This work was supported by National Natural Science Foundation of China (No. 71171162).

\section{REFERENCES}

[1] Mentrida-Pisano, J. A.; Tejero-Manzanares, J.; Aranguez-Ruiz, P.; Mata-Cabrera, F.; MontesTubio, F. (2015). Performance requirements for control and inspection entities in the regulatory field, DYNA, Vol. 90, No. 3, 250-253, doi:10.6036/7379

[2] Simon, D.; Snow, C. J.; Read, S. J. (2004). The redux of cognitive consistency theories: evidence judgments by constraint satisfaction, Journal of Personality and Social Psychology, Vol. 86, No. 6, 814-837, doi: 10.2139/ssrn.439984

[3] Glöckner, A.; Betsch, T. (2008). Modeling option and strategy choices with connectionist networks: Towards an integrative model of automatic and deliberate decision making, Judgment and Decision Making, Vol. 3, No. 3, 215-228, doi:10.2139/ssrn.1090866

[4] Yang, J.-P.; Zheng, J.-J. (2009). The effect of emotion on the quality of crisis decision-making, Acta Psychologica Sinica, Vol. 41, No. 6, 481-491, doi:10.3724/SP.J.1041.2009.00481

[5] Lu, J. T.; Yang, N. D.; Ye, J. F.; Wu, H. R. (2014). The influence paths of emotion on the occupational safety of rescuers involved in environmental emergencies - Systematic review article, Iranian Journal of Public Health, Vol. 43, No. 11, 1478-1485

[6] Lu, J. T.; Yang, N. D.; Ye, J. F.; Lei, T.; Mahmood, N. (2015). Differential effects of specific negative emotions on individual risk preference behaviors under social accidents: An analysis from the perspective of affective computing theory, Revista de Cercetare si Interventie Sociala, Vol. 50, 172-192

[7] DeKay, M. L.; Patinö-Echeverri, D.; Fischbeck, P. S. (2009). Better safe than sorry: Precautionary reasoning and implied dominance in risky decisions. Journal of Behavioral Decision Making, Vol. 22, No. 3, 338-361, doi:10.1002/bdm.630

[8] Hill, C.; Memon, A.; McGeorge, P. (2008). The role of confirmation bias in suspect interviews: A systematic evaluation, Legal and Criminological Psychology, Vol. 13, No. 2, 357-371, doi:10.1348/135532507X238682

[9] Pines, J. M. (2006). Profiles in patient safety: Confirmation bias in emergency medicine, Academic Emergency Medicine, Vol. 13, No. 1, 90-94, doi:10.1197/j.aem.2005.07.028

[10] Russo, J. E.; Carlson, K. A.; Meloy, M. G. (2006). Choosing an inferior alternative, Psychological Science, Vol. 17, No. 10, 899-904, doi:10.1111/j.1467-9280.2006.01800.x

[11] Jonas, E.; Graupmann, V.; Frey, D. (2006). The influence of mood on the search for supporting versus conflicting information: Dissonance reduction as a means of mood regulation?, Personality and Social Psychology Bulletin, Vol. 32, No. 1, 3-15, doi:10.1177/0146167205276118

[12] Read, S. J.; Simon, D.; Stenstrom, D. (2010). Hot cognitions in coherence-based reasoning and decision-making, $32^{\text {nd }}$ Annual Meeting of the Cognitive Science Society, 1465-1470

[13] Jonas, E.; Traut-Mattausch, E.; Frey, D.; Greenberg, J. (2008). The path or the goal? Decision vs. information focus in biased information seeking after preliminary decisions, Journal of Experimental Social Psychology, Vol. 44, No. 4, 1180-1186, doi:10.1016/j.jesp.2008.02.009

[14] Carlson, K. A.; Guha, A. (2011). Leader-focused search: The impact of an emerging preference on information search, Organizational Behavior and Human Decision Processes, Vol. 115, No.1, 133-141, doi:10.1016/j.obhdp.2010.12.002

[15] Bergert, F. B.; Nosofsky, R. M. (2007). A response-time approach to comparing generalized rational and take-the-best models of decision making, Journal of Experimental Psychology: Learning, Memory, and Cognition, Vol. 33, No. 1, 107-129, doi:10.1037/0278-7393.33.1.107

[16] Glöckner, A.; Betsch, T.; Schindler, N. (2010). Coherence shifts in probabilistic inference tasks, Journal of Behavioral Decision Making, Vol. 23, No. 5, 439-462, doi:10.1002/bdm.668

[17] Glöckner A.; Hodges, S. D. (2011). Parallel constraint satisfaction in memory-based decisions, Experimental Psychology, Vol. 58, No. 3, 180-195, doi:10.1027/1618-3169/a000084

[18] Glöckner, A.; Hilbig, B. E.; Jekel, M. (2014). What is adaptive about adaptive decision making? A parallel constraint satisfaction account, Cognition, Vol. 133, No. 3, 641-666, doi:10.1016/ j.cognition.2014.08.017 
[19] Simon, D.; Holyoak, K. J. (2002). Structural dynamics of cognition: From consistency theories to constraint satisfaction, Personality and Social Psychology Review, Vol. 6, No. 4, 283-294, doi:10.1207/s15327957pspr0604 03

[20] Svenson, O.; Salo, I.; Lindholm, T. (2009). Post-decision consolidation and distortion of facts, Judgment and Decision Making, Vol. 4, No. 5, 397-407

[21] McClelland, J. L.; Rumelhart, D. E. (1981). An interactive activation model of context effects in letter perception: I. An account of basic findings, Psychological Review, Vol. 88, No. 5, 375-407, doi: $10.1037 / 0033-295 X .88 .5 .375$

[22] Isen, A. M.; Patrick, R. (1983). The effect of positive feelings on risk taking: When the chips are down, Organizational Behavior and Human Performance, Vol. 31, No. 2, 194-202, doi:10.1016/ 0030-5073(83)90120-4

[23] Glöckner, A.; Betsch, T. (2008). Multiple-reason decision making based on automatic processing, Journal of Experimental Psychology: Learning, Memory, and Cognition, Vol. 34, No. 5, 10551075, doi:10.1037/0278-7393.34.5.1055

[24] Svenson, O. (1992). Differentiation and consolidation theory of human decision making: A frame of reference for the study of pre- and post-decision processes, Acta Psychologica, Vol. 80, No. 13, 143-168, doi:10.1016/0001-6918(92)90044-E

[25] Heugens, P. P. M. A. R.; Van Riel, C. B. M.; Van Den Bosch, F. A. J. (2004). Reputation management capabilities as decision rules, Journal of Management Studies, Vol. 41, No. 8, 13491377, doi: $10.1111 / \mathrm{j} .1467-6486.2004 .00478 . \mathrm{X}$

[26] Hao, Y.-H.; Yin, X.-L. (2014). The status seeking of decision maker in a work group and its effects on teamwork and team performance, Soft Science, Vol. 28, No. 8, 60-64, doi:10.3969/j.issn.1001-8409.2014.08.013

[27] Xia, X. H.; Liang, N. J. (2010). Effect of emotion and task types on information processing in purchasing decision-making, Psychological Research, Vol. 3, No. 2, 67-71

[28] Mairean, C.; Cimpoesu, D.; Turliuc, M. N. (2014). The effects of traumatic situations on emergency medicine practitioners, Revista de Cercetare si Interventie Sociala, Vol. 44, 279-290

[29] Schwartz, N.; Clore, G. L. (1983). Mood, misattribution, and judgments of well-being: Informative and directive functions of affective states, Journal of Personality and Social Psychology, Vol. 45, No. 3, 513-523, doi: $\underline{10.1037 / 0022-3514.45 .3 .513}$ 